\title{
DREADDing proglucagon neurons: a fresh look at metabolic regulation by the brain
}

\author{
Jonathan E. Campbell and David A. D'Alessio \\ Division of Endocrinology, Duke University, Durham, North Carolina, USA.
}

\begin{abstract}
Glucagon-like peptide 1 receptor (CLP-1R) signaling in the CNS has been linked to reduced food intake, lower body weight, improved glucose homeostasis, and activation of CNS stress axes. GLP-1 is produced by cells that express proglucagon (CCC); however, the stimuli that activate GCC neurons are not well known, which has made understanding the role of this neuronal population in the CNS a challenge. In this issue of the JCI, Gaykema et al. use designer receptors exclusively activated by designer drugs (DREADD) technology to specifically activate GCG+ neurons in mouse models. While activation of GCC ${ }^{+}$neurons did reduce food intake, and variably decreased hepatic glucose production, other CLP-1-associated effects were not observed - e.g., activation of stress axes or stimulation of insulin secretion - in response to GCG+ neuron activation. The authors have provided a valuable model to study this set of neurons in vivo, and their results provide new insights into the function of GCG $^{+}$neural activity in the brain and raise questions that will move research on this clinically relevant neural system forward.
\end{abstract}

Given the high concentration of GLP$1 \mathrm{R}$ in the hypothalamus, it is not surprising that many of the responses attributed to GLP-1 in the CNS are related to energy and nutrient metabolism. GLP-1R activation in specific areas of the brain potently decrease food intake, either as a consequence of engaging neuronal pathways that increase satiety or by activating neurons that lead to aversion or malaise. Chronic administration of GLP-1R agonists directly to the CNS decreases body weight in animal models in conjunction with decreased caloric consumption (5). In addition to the effects on body weight, there is evidence that GLP-1 regulates glucose homeostasis via neuronal pathways that enhance insulin secretion, decrease hepatic glucose output, and increase glucose disposal into skeletal muscle $(6,7)$. Finally, GLP-1 stimulates two different stress axes in the CNS - the hypothalamic-pituitary-adrenal (HPA) axis and the sympathetic nervous system (SNS) thereby causing elevations in circulating corticosterone and epinephrine.

\section{Activating Gcg-expressing neurons with DREADDs}

The stimuli that activate hindbrain $G c g$ expressing neurons are not well established and have been difficult to ascertain in controlled experiments. Thus, understanding the physiology of GCG-positive neurons has depended entirely on specific gain- and loss-of-function studies of GLP$1 \mathrm{R}$ signaling in the CNS. In this issue (8), Gaykema and colleagues present a method that they devised for interrogating the brain GLP-1 system. Their approach involves using designer receptors exclusively activated by designer drugs (DREADD) technology to activate $G c g$-expressing neurons. Stereotaxic administration of an adeno-associated virus (AAV) into the caudal medulla delivered a Gaq-coupled construct that is expressed in the presence of Cre recombinase. Expression of the Gaq-coupled DREADD (hM3Dq) 


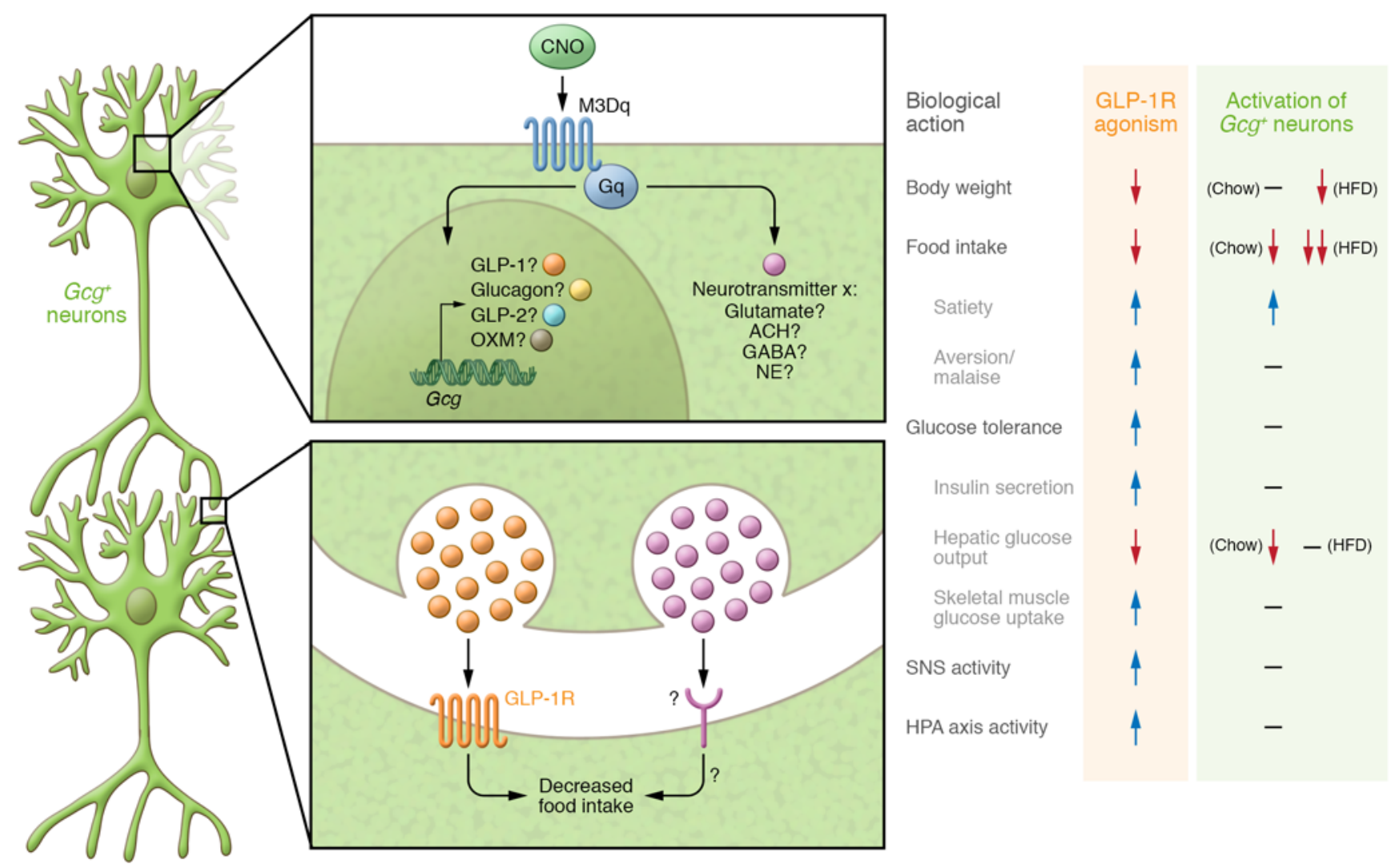

Figure 1. Comparison of the physiological effects of activating GCG+ neurons versus peripheral or systemic activation of GLP-1R. Clozapine N-oxide (CNO) activation of Gaq receptors expressed exclusively in GCG+ neurons leads to a decrease in food intake. The neurotransmitter responsible, as well as the projection sites of the GCG+ neurons, is unclear. Support for GLP-1 as a key neurotransmitter secreted by GCG ${ }^{+}$neurons is provided by the idea that GLP-1 is likely a product of the cells and also that GCG+ neurons project on to GLP-1R+ cell bodies in the hypothalamus, a key CNS site that controls many of the physiological actions outlined here. However, the fact that activation of GCG+ neurons fails to elicit previously demonstrated effects of CNS GLP-1 leaves open the question as to which neurotransmitter these cells express. It still remains to be seen whether these discrepancies arise due to differences in methodology (i.e., physiology versus pharmacology) or if activation of GCG+ neurons provides more precise activation of biological events (i.e., only food intake). OXM, oxyntomodulin; Ach, acetylcholine; NE, norepinephrine.

in $\mathrm{Gcg}$-Cre mice resulted in selective expression of this receptor in $\mathrm{GCG}^{+}$neurons, which could then be activated with clozapine $\mathrm{N}$-oxide (CNO). This system proved to be both specific and efficacious for activating $\mathrm{GCG}^{+}$neurons with $\mathrm{CNO}$, as evidenced by both colocalization of Fos and GLP-1, as well as electrophysiological recordings of neuronal firing following CNO administration. Remarkably, activation of $\mathrm{GCG}^{+}$neurons failed to engage many of the previously defined actions of GLP-1R agonism in the CNS (Figure 1). Acute treatment with $\mathrm{CNO}$ did not impact insulin levels or skeletal muscle glucose uptake. And while CNO modestly decreased gluconeogenesis in mice on a standard rodent diet, this effect did not translate into a measurable impact on glucose tolerance. Furthermore, $\mathrm{CNO}$ administration did not engage the HPA axis, elevate SNS activity, or change anx- iety behaviors, all of which have been noted in previous studies of GLP-1R stimulation in the CNS. The most apparent effect on $\mathrm{GCG}^{+}$neuron activation was a robust decrease in food intake, which was attributed to an increase in satiety rather than malaise. Chronic $\mathrm{GCG}^{+}$neuron activation failed to induce changes in body weight in mice on a standard diet. Interestingly, decreased food intake was magnified when the experiment was repeated in mice on a high-fat diet (HFD), to the extent that chronic $\mathrm{CNO}$ treatment caused a decrease in body weight. This interaction of diet and $\mathrm{GCG}^{+}$neuron activation seems specific to food intake, as the stress responses were not induced this experiment, and reduction of hepatic glucose output in response to CNO was lost.

The results of Gaykema and coworkers (8) add a new dimension to understanding the role of proglucagon peptides in the brain, a topic of continued translational importance, as new drugs that are co-agonists of both GLP-1R and glucagon receptors are being explored as treatments for metabolic diseases. The DREADD approach allows the discrete $\mathrm{GCG}^{+}$neural system to be activated from the bottom up, presenting a novel view of this system's capacity. Testing the Gcg-expressing neural system in the context of what is known from studies with CNS administration or blockade of GLP-1R is appropriate in that this ligand/receptor pathway has been extensively mapped, with a list of responses defined (Figure 1). The recapitulation of some, but not all, of the effects previously attributed to GLP-1 in the CNS raises interesting questions that should drive the field forward. On one hand, effects of brain GLP-1 on glucose control and on HPA and SNS activation may be pharmacologic responses that are not mediated by 
normal neural function. On the other, in order to fully interpret these findings more details and validation of the $G c g$-DREADD model are needed, including whether activation by CNO achieves the full range of physiologic neural activation and what factors are released from $\mathrm{GCG}^{+}$nerves. In fact, based on the data presented, it can only be assumed that the effect of $\mathrm{CNO}$ to reduce food intake involves GLP-1, or $G c g$-associated products. Combining the $G c g$-DREADD model with CNS administration of GLP-1R or GCG receptor antagonists could provide clarity here. Moreover, it remains possible that food intake is the most sensitive response to $\mathrm{GCG}^{+}$neural activation, with stress and illness responses occurring only when the system is firing at near maximal rates. Nonetheless, the $G c g$-DREADD model provides a firm base from which to address a tractable neural system that likely plays a significant role in systemic metabolism.

\section{Future considerations}

Some of the findings of Gaykema et al. (8) support their contention that targeting $\mathrm{GCG}^{+}$neurons might have distinct potential for therapeutic development. For example, it would be very advantageous if activation of these nerves can cause satiety without malaise or visceral illness, since nausea is currently the limiting side effect of GLP-1R agonists in clinical use. It is also interesting to consider whether targeting $\mathrm{GCG}^{+}$neurons might truly alleviate activation of stress responses seen with GLP-1R agonists. This could provide greater or more lasting weight loss, as CNS administration of GLP-1 to animals (9) and peripheral GLP1 administration to humans (10) increase circulating glucocorticoids as well as satiety, responses with opposing impacts on body weight. While these examples are speculative at present, they provide an impetus for a better understanding of
$\mathrm{GCG}^{+}$neurons and their physiologic and pharmacologic activation.

One of the most interesting findings in these studies is the enhanced effect of $\mathrm{GCG}^{+}$neuron activation on anorexia and weight loss in obese mice. These findings are consistent with previous work showing potentiation of GLP-1 effects on feeding by leptin (11), a peptide increased in obesity. This interaction suggests a role for GLP-1 to mitigate the effects of metabolic stress in the CNS, potentially as a mechanism to restore homeostasis. A similar hypothesis has been proposed for understanding the role of GLP-1 produced in other tissues. $\alpha$ Cell production of GLP-1 is minimal in healthy rodent and human islets yet increases dramatically in the face of stressors such as proinflammatory cytokines (12) or streptozotocin (13). Hence, GLP-1 produced in $\alpha$ cells is situated to respond to metabolic stress by stimulating $\beta$ cell function and promoting glucose homeostasis. In the gut, both IL-6 and bacterial endotoxin stimulate GLP-1 from L cells, and GLP-1Rs in the intestine are located on intraepithelial lymphocytes (14), suggesting a mucosal GLP-1 system that is responsive to environmental stress. This broad framing of GLP-1 actions may provide some predictive utility in understanding $G c g$-encoded peptides in the three regions where they are produced. The contribution of Gaykema and colleagues presented here provides a tangible means to move ahead in the study of the least-understood of these regions, the brain.

Address correspondence to: David D'Alessio, Duke University Division of Endocrinology, Duke Molecular Physiology Institute, 300 N. Duke St., Durham, North Carolina 27701, USA. Phone: 919.684 .5778 ; E-mail: david.d'alessio@duke.edu.

1. Drucker DJ, Nauck MA. The incretin system: glucagon-like peptide-1 receptor agonists and dipeptidyl peptidase- 4 inhibitors in type 2 diabetes. Lancet. 2006;368(9548):1696-1705.

2. Sandoval DA, D'Alessio DA. Physiology of proglucagon peptides: role of glucagon and GLP-1 in health and disease. Physiol Rev. 2015;95(2):513-548.

3. Cork SC, Richards JE, Holt MK, Gribble FM, Reimann F, Trapp S. Distribution and characterisation of Glucagon-like peptide-1 receptor expressing cells in the mouse brain. Mol Metab. 2015;4(10):718-731.

4. Heppner KM, et al. Expression and distribution of glucagon-like peptide-1 receptor mRNA, protein and binding in the male nonhuman primate (Macaca mulatta) brain. Endocrinology. 2015;156(1):255-267.

5. Barrera JG, Sandoval DA, D'Alessio DA, Seeley RJ. GLP-1 and energy balance: an integrated model of short-term and long-term control. Nat Rev Endocrinol. 2011;7(9):507-516.

6. Knauf C, et al. Brain glucagon-like peptide-1 increases insulin secretion and muscle insulin resistance to favor hepatic glycogen storage. J Clin Invest. 2005;115(12):3554-3563.

7. Sandoval DA, Bagnol D, Woods SC, D'Alessio DA, Seeley RJ. Arcuate glucagon-like peptide 1 receptors regulate glucose homeostasis but not food intake. Diabetes. 2008;57(8):2046-2054.

8. Gaykema RP, et al. Activation of murine preproglucagon-producing neurons reduces food intake and body weight. J Clin Invest. 2017;127(3):1031-1045.

9. Kinzig KP, et al. CNS glucagon-like peptide-1 receptors mediate endocrine and anxiety responses to interoceptive and psychogenic stressors. J Neurosci. 2003;23(15):6163-6170.

10. Vella A, et al. Effect of glucagon-like peptide-1(736)-amide on initial splanchnic glucose uptake and insulin action in humans with type 1 diabetes. Diabetes. 2001;50(3):565-572.

11. Williams DL, Baskin DG, Schwartz MW. Leptin regulation of the anorexic response to glucagon-like peptide-1 receptor stimulation. Diabetes. 2006;55(12):3387-3393.

12. Ellingsgaard $H$, et al. Interleukin- 6 enhances insulin secretion by increasing glucagon-like peptide-1 secretion from $L$ cells and alpha cells. Nat Med. 2011;17(11):1481-1489.

13. Nie Y, et al. Regulation of pancreatic PC1 and PC2 associated with increased glucagon-like peptide 1 in diabetic rats. J Clin Invest. 2000;105(7):955-965.

14. Yusta B, et al. GLP-1R agonists modulate enteric immune responses through the intestinal intraepithelial lymphocyte GLP-1R. Diabetes. 2015;64(7):2537-2549. 